JOURNAL OF

APPLIED

CRYSTALLOGRAPHY

ISSN 1600-5767

Keywords: book reviews; electrons in solids.

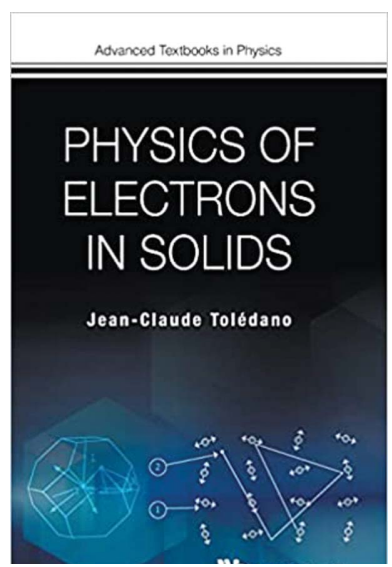

\section{Physics of Electrons in Solids. By Jean-Claude Toledano. World Scientific, 2021. Pp. 320. Hardback price GBP 95.00. ISBN 978-1-78634-972-9.}

\author{
Mike Glazer*
}

University of Oxford, Department of Physics, Parks Road, Oxford OX1 3PU, United Kingdom. *Correspondence e-mail: mike.glazer@physics.ox.ac.uk

I like the way that the author Jean-Claude Toledano begins his book with 'The content of this textbook can be considered as an elaborate answer to the following question: why does copper, the metal commonly used in electrical connections, possess a value of electrical resistivity as low as $1.6 \mu \Omega \mathrm{cm}$ at room temperature.' Furthermore, he states that he will assume that electrons are the only constituents of a solid. The atomic cores are therefore ignored, at least in the first place, but they manifest their presence through an electrostatic potential acting on the electrons. Now, these are bold statements, but welcome nonetheless, since they lay out for the reader what to expect. In this way, he sets the scene for what follows through 11 chapters.

The level of this textbook, I would say, is that of an advanced undergraduate or graduate student. A useful feature of the book is the way each chapter begins with a paragraph detailing the main ideas, so that you have an idea in advance of what to expect. Unlike most condensed matter physics (CMP) books, the stress is mainly on the quantum aspects of the subject. Having said that, the author does go on to explain over several chapters the usual concepts used in crystallography, although in a slightly unusual way which may irritate crystallographic purists. As such, the approach is somewhat oldfashioned. Let me give a brief report on each chapter.

Chapter 1 introduces the idea that solids can be thought of as quantum systems and is an easy read. Chapter 2, entitled The crystalline order, explains the usual concepts of lattices, unit cells and space groups, although not in much detail. Toledano defines the structure of a solid as the 'configuration in space' of the constituting atoms. He does qualify this in a footnote to take into account the vibrational motion of the atoms, and therefore his definition refers to average positions. He mentions that there are 230 space symmetries of crystals but does not mention the use of the term 'type' in the modern sense. Although he is careful to distinguish between lattice and structure, I fear that some statements such as 'this lattice can be considered as part of the structure' could confuse the novice student. One of the bits of terminology that I, personally, found irritating is his use of 'simple' instead of 'primitive', such as in 'simple cubic' or 'simple Bravais lattices'. Unfortunately, I know that most physics texts use this terminology, so who am I to object? When talking about cubic lattices, the word 'type' is employed to refer to the different conventional choices of unit cell (primitive, face-centred cubic and body-centred cubic), rather than to express that while there are 14 Bravais lattice 'types', there are an infinite number of Bravais lattices. The description of structures is made using the usual lattice and basis method, which works fine for very simple structures and is found in just about all CMP books. For physics students, this is the usual way to introduce lattice and structure. One passage made me have to think. This where Toledano says that there are only seven possible symmetry groups of Bravais lattices, associated with the 'shapes' of the Bravais lattices. Actually, a lattice does not have a shape; the unit cell used to describe it does. The correspondence with the symmetry is correct only if the conventional unit cell is adopted.

The next chapter is called The reciprocal space as a space of quantum numbers. This is where we meet the Bloch wave-form and the reciprocal lattice. The importance of the Wigner-Seitz cell and the Brillouin zone is emphasized. An interesting point made is that imposing a finite total probability $(p=1)$ in an infinitely extended crystal implies the nonphysical condition of a vanishingly small probability in each cell. Conversely, a finite probability in each cell determines an infinite probability in the entire crystal. I had never 
thought of that before, but that is the joy of always learning new ideas. The chapter ends with an appendix acting as a reminder of quantum mechanics. I am not sure that this is necessary, as any student approaching this book should have already acquired a good knowledge of quantum mechanics.

Chapter 4 then discusses The reciprocal space as a space of diffraction patterns. Here, Toledano explains the usual diffraction conditions and how these relate to the reciprocal lattice, the Bragg and Laue equations, and the Ewald sphere construction. He gives a brief description of photographic methods to record diffraction information (precession and powder), but nothing on modern diffractometers. We read in a small section on electron diffraction the surprising statement that electron diffraction is not, in general, a suitable method for determining the configuration of atoms in the structure. This is way out of date, because recent advances in detector designs and methods of collecting electron diffraction data do enable atomic configurations to be determined. Even the longheld belief that multiple scattering is a problem has been reasonably successfully dealt with.

Chapter 5 is on the quantum states of an electron in a crystal. This is where energy band gaps determined via perturbation theory are discussed, followed by the tightbinding approximation. This is a thorough treatment that is not usually found to this level in standard CMP books. Chapter 6 is on equilibrium electronic properties of solids, in which Toledano introduces the Fermi level and energy bands, very traditional in CMP textbooks. In Chapter 7, the discussion turns to the dynamics of electrons in crystals, and Chapter 8 is on electronic transport properties. The Drude model, which is essentially a classical theory, is discussed, explaining its limitations and how quantum theory is needed. Chapter 9 deals with intrinsic and doped semiconductors using the band structures of silicon and gallium arsenide: all standard material in a CMP text.

Chapter 10 is on solids as systems of particles in interaction. The Born-Oppenheimer approximation is explained as well as the Hartree solution. Then, Chapter 11 ends with a relatively brief explanation of ferromagnetism and superconductivity. The Landau theory of phase transitions (not explicitly named as such) is used to obtain the Curie law. The reason for ferromagnetism to occur at normal temperatures is explained in terms of the exchange energy between two fixed ions. In most books, this subject is tackled rather in terms of a molecular field approach.

The book ends with some exercises. These are quite difficult and the solutions that follow are welcome.

In summary, therefore, my verdict on this book is that it is a useful text for students, although it is let down by a few unfortunate matters. Many of the diagrams are rather crude or too complicated in my view and could be improved (perhaps in the next edition?). There are several mistakes in the use of English language that could be dealt with easily by consulting a native English speaker. Instances are words like intersticial, unequivalent, alcaline, somme (instead of some) and the phrase ... quantum argument infirms the validity....

Despite these rather minor quibbles and sometimes oldfashioned approaches, especially in crystallography, I do recommend this book. It was a pleasure to read, and I learnt a number of things that I had not appreciated before. 